\title{
Search for possibly evolutionary linked globular and open clusters
}

\author{
V.V. Bobylev'1 and A.T. Bajkova \\ Main (Pulkovo) Astronomical Observatory, Russian Academy of Sciences, \\ St.-Petersburg, 196140 Russia
}

\begin{abstract}
Based on a large sample of 133 Galactic globular clusters we obtained a new estimate of the frequency of globular-cluster impacts onto the Galactic plane, which we found to be equal to three events per 1 Myr. Our computations involving new kinematical data do not support the well-known hypothesis about the possible origin of the open cluster Stephenson 2 as a result of the massive globular cluster $\omega$ Cen crossing the Galactic disk. Our results also do not support the well-known hypothesis that the globular cluster NGC6397 could trigger the formation of the open cluster NGC6231. We found for the first time six globular clusters, which could have triggered the formation of an open cluster when crossing the Galactic plane. These are the globular clusters NGC104, NGC2808, NGC6362, NGC6540, NGC6749, and NGC6752. For each of these clusters we identify one or several open clusters, which were possibly born via such scenario. In our opinion, of greatest interest are the pairs NGC104-Ruprecht 129, and NGC6362-Pismis 11.
\end{abstract}

\section{INTRODUCTION}

The crossing of the Galactic disk by a globular cluster (GC) can stimulate star formation processes. This process may involve (a) gravitational focusing where as a result of the approach of the GC to the disk matter is pulled to a certain point and/or (b) strong contraction of the disk matter in a certain direction. In the latter case the strongest contraction effect is observed in the case of the oblique incidence of a massive body, as established by Comeron and Torra [1, 2] who modeled an impact of a massive high-velocity cloud onto the Galactic disk. Furthermore, the computations performed by Levy [3] showed that the passage of GC through the Galactic disk may produce a shock wave. Wallin et al. [4] showed, based on the model of the gravitational focusing of the gas, that large OB-associations may form after about 30 Myr after the GC passage through the Galactic disk.

Brosche et al. [5] appear to be the first to raise the issue about finding some observational consequences of the Galactic-disk crossings of two GCs, NGC362 and NGC6218. For example, the cluster NGC362 should have crossed the disk at rather high velocity for mass ejection from the disk to be possible.

To consider another scenario, Rees and Cudworth [6] proposed to study the NGC6397NGC6231 pair (a GC and a young open cluster). The above authors associated the Galacticdisk crossing by the GC with the subsequent formation of the open star cluster (OSC) at the crossing place.

\footnotetext{
${ }^{1}$ e-mail: vbobylev@gaoran.ru
} 
Vande Putte and Cropper [7] analyzed the Galactic trajectories of 54 GCs with measured proper motions, radial velocities, and distances and found the NGC3201, NGC6397, and NGC6838 clusters to be the promising sites for searching. Near the crossing places of these GCs OB-associations are located, which could have formed as a result of the impact of a $\mathrm{GC}$ onto the disk. Salerno et al. [8] showed that the passage of the GC $\omega$ Cen through the Galactic disk could have triggered the formation of the OSCs Stephenson 2 and [BDS2003] 122.

GCs may be involved not only in the formation of OSCs, but also in their destruction. De la Fuente Marcos et al. [9] computed the parameters of mutual encounters between known Milky Way globular and open clusters. The record breaker turned out to be the pair consisting of the GC FSR 1767 (2MASS-GC04) and young OSC Ruprecht 127: their separation 22 million years ago was only 36 pc. The above authors consider this pair as an example of the destruction of clusters because they come too close to each other.

The results of such studies are very much dependent on the measurement errors of the observed cluster parameters, in particular, on the errors of their proper motions. Therefore the publication of high precision proper motions for a number of Milky-Way GCs based on Hubble Space Telescope observations [10-12] served as a stimulus for carrying out this work.

The aim of this study is

- To estimate the frequency of the passages of GCs across the Galactic midplane by numerically integrating orbits in the most realistic model of the Galactic potential taking into account the contribution of both axisymmetric components (bulge, disk, and halo) and that of the bar and spiral structure with the parameters determined from the most recent observational data;

- Validate some of the well-known results of various authors;

- And, most importantly, use modern data to search for other possible cases of the formation of OSCs in the Galactic disk triggered by the passage of a GC through it.

\section{THE DATA}

Our cluster sample is based on the MWSC (Milky Way Star Clusters) catalog presented in [13]. It contains the positions, proper motions, and radial velocities for stars both in open and globular clusters of our Galaxy. When analyzing the positions of OSCs we use more extensive catalog [14]. We usually excluded objects marked by labels "a", "n", and "s" in these catalogs (associations, nebulae, and asterisms).

The average proper motions of clusters in the MWSC are based on the stellar proper motions of PPMXL catalog [15], which have large random errors ranging from 4 to 10 mas $\mathrm{yr}^{-1}$. One of the aims of this paper is to construct the Galactic orbits of globular and open clusters over long time intervals. This requires maximum possible accurate knowledge of the initial kinematic parameters. For this reason we adopted more reliable data for a number of GCs.

1. We used the data from [11], where the mean absolute proper motions of ten selected Galactic bulge GCs are derived from both ground-based observations made with European Southern Observatory telescopes and from the data obtained with the Hubble Space Telescope (HST). The average epoch difference was 25 years and the average error of the inferred

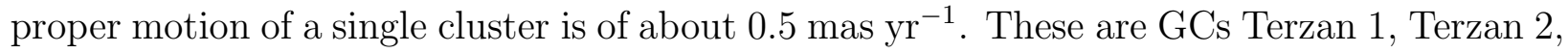
Terzan 4, Terzan 9, NGC6522, NGC6540, NGC6558, NGC6652, NGC6681, and Palomar 6. 
A detailed analysis of their individual orbits can be found in [16]. For clusters Terzan 2, Terzan 4, NGC6652, and Palomar 6 we applied corrections in accordance with [17].

2. We adopted the absolute proper-motion components and heliocentric distance to $\omega$ Cen cluster inferred from HST observations and reported in [12]:

$$
\begin{array}{r}
\mu_{\alpha} \cos \delta=-3.238 \pm 0.028 \operatorname{mas}_{\mathrm{yr}^{-1}} \\
\mu_{\delta}=-6.716 \pm 0.043 \mathrm{mas}^{-1} \\
r=5.20 \pm 0.25 \mathrm{kpc}^{2}
\end{array}
$$

The proper-motion components determined in (1) differ strongly from those used in [8]

$$
\begin{array}{r}
\mu_{\alpha} \cos \delta=-5.08 \pm 0.35 \text { mas } \mathrm{yr}^{-1} \\
\mu_{\delta}=-3.57 \pm 0.34{\mathrm{mas} \mathrm{yr}^{-1}}^{r}=5.3 \pm 0.5 \mathrm{kpc}
\end{array}
$$

when modeling the passage of the $\omega$ Cen cluster through the Galactic disk. Therefore

repeating the study carried out in [8], which we perform in this paper using new data and a different model of Galactic gravitational potential is of great interest.

3.We use the proper motions of theGCsNGC104, NGC5272, NGC6121, NGC6397, and NGC6656 based on Gaia TGAS (Tycho-Gaia Astrometric Solution) catalog data by Watkins and van den Marel [18]. The above authors also report the average trigonometric parallaxes for the five clusters. However, it is their proper motions that are of primary importance. The distance estimates are not too precise because they are based on the few stars in each cluster. Furthermore, distances greater than $2 \mathrm{kpc}$ so far cannot be reliably determined because of the parallax errors in the current GaiaDR1 release.

4. To complete the picture, we point out our use of new measurements of the components of the absolute proper motion of the NGC2419 cluster: $\left(\mu_{\alpha} \cos \delta, \mu_{\delta}\right)=(-0.17 \pm 0.26 ;-0.49 \pm$ 0.17) mas $_{\mathrm{yr}^{-1}}$, based on HST and GaiaDR1 data by Massari et al. [19]. These values differ strongly from those reported in catalog [13].

5. NGC6397 is one the closest GCs to the Sun. As we pointed out in item 3 of this section, we use for this cluster the new precise proper motion values from [18]. Furthermore, Brown et al. [20] recently found a new estimate for the trigonometric parallax of this cluster, $\pi_{\text {trig }}=0.418 \pm 0.018$ mas, based on HST observations. As a result, we can now test the hypothesis of Rees and Cudworth [6] at a new level of accuracy.

\section{THE METHOD}

The method that we use to search OSCs whose formation was likely triggered by the impact of GC onto the Galactic disk is based on integration of the orbits of globular and open clusters in the Galactic gravitational potential. Integration of GCs orbits allow us to determine coordinates and times of the passage of the GC through the Galactic disk. Integration of OSCs orbits over a certain interval allows us to determine how close they were to the reconstructed impact sites and likely time of the birth of the OSC. The use of statistical Monte Carlo method makes it possible to construct an entire domain $(X, Y)$ of globularcluster crossings with the Galactic disk and also the set of orbits of the OSCs considered taking into account the errors in the kinematical data of objects and parameters of the Galactic model. This makes it possible to estimate the degree of overlap of globular-cluster impact domains with open-cluster orbits and hence the probability of the birth of an OSC. 
Note that in all numerical simulations performed within the framework of this paper we used 1000 Monte-Carlo iterations to construct the confidence domain of the points where the GC crosses the Galactic disk and 100 iterations when constructing the ensemble of open-cluster orbits. The MWSC catalog does not give the random errors of the inferred cluster distances and we set them equal to $10 \%$ (except otherwise stated) for all clusters of the this catalog. Note that the confidence intervals of the reconstructed sets of (1) points where the GC intersect with the Galactic plane and (2) open-cluster orbits correspond to the probability of $99.7 \%(3 \sigma)$.

We describe our adopted model of the Galaxy below. We consider the Navarro-Frank-White model [23] that we refined based on modern data [21, 22] and extended with terms taking into account the effect of the central bar and spiral density wave to be most realistic.

\section{Model of Axisymmetric Potential of the Galaxy}

Model of the axisymmetric gravitational potential of the Galaxy has the form of the sum of three components - central spherical bulge $\Phi_{b}(r(R, Z))$, disk $\Phi_{d}(r(R, Z))$, and massive spherical dark-matter halo $\Phi_{h}(r(R, Z))$ :

$$
\Phi(R, Z)=\Phi_{b}(r(R, Z))+\Phi_{d}(r(R, Z))+\Phi_{h}(r(R, Z)) .
$$

Here we use cylindrical coordinate system $(R, \psi, Z)$ with the origin at the Galactic center. In the Cartesian coordinate system $(X, Y, Z)$ with the origin at the Galactic center the distance to a star (spherical radius) is $r^{2}=X^{2}+Y^{2}+Z^{2}=R^{2}+Z^{2}$. The $X$-axis points from the Galactic center to the Sun; the $Y$-axis is perpendicular to the $X$-axis and points in the direction of Galactic rotation, and the $Z$-axis is perpendicular to the Galactic plane $X Y$ toward the North Galactic pole. The gravitational potential is given in the units of $100 \mathrm{~km}^{2} \mathrm{~s}^{-2}$, distances are in $\mathrm{kpc}$, and masses in the units of the mass of the Galaxy, $M_{\text {gal }}=2.325 \times 10^{7} M_{\odot}$, corresponding to Galactic constant $G=1$.

The potentials of the bulge $\Phi_{b}(r(R, Z))$ and disk $\Phi_{d}(r(R, Z))$ have the form proposed by Miyamoto and Nagai [24]:

$$
\begin{gathered}
\Phi_{b}(r)=-\frac{M_{b}}{\left(r^{2}+b_{b}^{2}\right)^{1 / 2}}, \\
\Phi_{d}(R, Z)=-\frac{M_{d}}{\left[R^{2}+\left(a_{d}+\sqrt{Z^{2}+b_{d}^{2}}\right)^{2}\right]^{1 / 2}},
\end{gathered}
$$

where $M_{b}, M_{d}$ are the masses of the components and $b b, a d, b d$ are the scale parameters in kpc. The halo component is adopted in the form of the Navarro-Frank-White model [23]:

$$
\Phi_{h}(r)=-\frac{M_{h}}{r} \ln \left(1+\frac{r}{a_{h}}\right) .
$$

Table 1 lists the values of the parameters of Galactic potential model (4)-(6) found in $[21,22]$ based on circular rotation velocities of Galactic objects located at distances out to $R \sim 200 \mathrm{kpc}$. Note that the corresponding Galactic rotation curve was constructed adopting the solar Galactocentric distance of $R_{0}=8.3 \mathrm{kpc}$ and LSR velocity relative to the Galactic center $V_{0}=244 \mathrm{~km} \mathrm{~s}^{-1}$. 
Table 1: Parameters of the Galactic potential model $M_{\text {gal }}=2.325 \times 10^{7} M_{\odot}$

\begin{tabular}{|c|c|}
\hline Parameters & Values \\
\hline$M_{b}\left(\mathrm{M}_{\text {gal }}\right)$ & $443 \pm 27$ \\
$M_{d}\left(\mathrm{M}_{\text {gal }}\right)$ & $2798 \pm 84$ \\
$M_{h}\left(\mathrm{M}_{\text {gal }}\right)$ & $12474 \pm 3289$ \\
$b_{b}(\mathrm{kpc})$ & $0.2672 \pm 0.0090$ \\
$a_{d}(\mathrm{kpc})$ & $4.40 \pm 0.73$ \\
$b_{d}(\mathrm{kpc})$ & $0.3084 \pm 0.0050$ \\
$a_{h}(\mathrm{kpc})$ & $7.7 \pm 2.1$ \\
\hline
\end{tabular}

\section{Taking into Account the Bar and Galactic Spiral Pattern}

In the case of the Galactic bar and spiral pattern the corresponding terms describing the potential of these components are added to the right-hand side of formula (3). We adopted the bar potential in the form of the triaxial ellipsoid model in accordance with [25]:

$$
\Phi_{B}=-\frac{M_{B}}{\left(q_{B}^{2}+X^{2}+\left[Y \cdot a_{B} / b_{B}\right]^{2}+\left[Z \cdot a_{B} / c_{B}\right]^{2}\right)^{1 / 2}},
$$

where $M_{B}$ is the mass of the bar, which is equal to $43.1 \times \mathrm{M}_{g a l} ; a_{B}, b_{B}$, and $c_{B}$ are the three semiaxes of the bar $\left(a_{B} / b_{B}=2.381, a_{B} / c_{B}=3.03\right) ; q_{B}$ is the length of the bar; $X=R \cos \vartheta$ and $Y=R \sin \vartheta$, where $\vartheta=\theta-\Omega_{B} \cdot t-\theta_{B}, \theta$ is the initial position angle of the object: $\tan \theta=Y_{0} / X_{0}\left(X_{0}, Y_{0}\right.$ are the initial coordinates of the object in the Cartesian coordinate system in accordance with (11)), $\Omega_{B}$ is the circular velocity of the bar, $t$ is time, $\theta_{B}$ is the bar orientation angle relative to Galactic axes $X, Y$, which is counted from the line connecting the Sun and the Galactic center (the $X$-axis) to the major axis of the bar in the direction of Galactic rotation. We adopted the estimates of the bar parameters $\Omega_{B}=55 \mathrm{~km} \mathrm{~s}^{-1} \mathrm{kpc}^{-1}$, $q_{B}=8 \mathrm{kpc}$, and $\theta_{B}=45^{\circ}$ from [26].

The potential of spiral density wave $[27,28]$ is described by the following formula [29]:

$$
\Phi_{s p}(R, \theta, t)=A \cos \left[m\left(\Omega_{p} t-\theta\right)+\chi(R)\right]
$$

where

$$
A=\frac{\left(R_{0} \Omega_{0}\right)^{2} f_{r 0} \tan i}{m}, \quad \chi(R)=-\frac{m}{\tan i} \ln \left(\frac{R}{R_{0}}\right)+\chi_{\odot},
$$

Here $A$ is the amplitude of the spiral-wave potential; $f_{r 0}$ is the ratio of the radial component of the perturbation from spiral arms to the general gravitational attraction of the Galaxy; $\Omega_{p}$ is the angular velocity of rigid rotation of the wave; $m$, the number of spiral arms; $i$, the pitch angle of spiral arms ( $i<0$ for trailing arms); $\chi$ is the phase of the radial wave and in that case $\chi=0^{\circ}$ corresponds to the arm center; $\chi_{\odot}$ is the radial phase of the Sun with respect to the spiral arm. We adopted the following values for the parameters of the spiral wave:

$$
\begin{aligned}
& m=4, \\
& i=-13^{\circ}, \\
& f_{r 0}=0.05, \\
& \chi_{\odot}=-120^{\circ}, \\
& \Omega_{p}=20 \mathrm{~km} \mathrm{~s}^{-1} \mathrm{kpc}^{-1} .
\end{aligned}
$$




\section{Equations of Motion}

Equations of motion of a test particle in the Galactic potential have the following form:

$$
\begin{aligned}
& \dot{X}=p_{X}, \quad \dot{Y}=p_{Y}, \quad \dot{Z}=p_{Z} \\
& \dot{p}_{X}=-\partial \Phi / \partial X \\
& \dot{p}_{Y}=-\partial \Phi / \partial Y \\
& \dot{p}_{Z}=-\partial \Phi / \partial Z
\end{aligned}
$$

where $p_{X}, p_{Y}$, and $p_{Z}$ are the canonical moments and dot denotes differentiation with respect to time. We integrate equations (10) using the fourth-order Runge-Kutta algorithms.

We set the peculiar velocity of the Sun relative to the LSR equal to $\left(u_{\odot}, v_{\odot}, w_{\odot}\right)=$ $(11.1,12.2,7.3) \mathrm{km} \mathrm{s}^{-1}$, in accordance with [30]. Here we give the heliocentric velocities measured in a moving Cartesian coordinate system: $u$ points toward the Galactic center $v$ points toward Galactic rotation, and $w$ is perpendicular to the Galactic plane in the direction toward North Galactic pole.

Let us now denote the initial positions and spatial velocities of test particles in the heliocentric coordinate system as $\left(x_{o}, y_{o}, z_{o}, u_{o}, v_{o}, w_{o}\right)$. Then in the Galactic Cartesian coordinate system the initial values of the positions and velocities of test particles are given by the following formulas:

$$
\begin{aligned}
& X_{0}=R_{0}-x_{o}, \quad Y_{0}=y_{o}, \quad Z_{0}=z_{o}+h_{\odot} \\
& U=-\left(u_{o}+u_{\odot}\right) \\
& V=v_{o}+v_{\odot}+V_{0} \\
& W=w_{o}+w_{\odot}
\end{aligned}
$$

where $h_{\odot}=16 \mathrm{pc}$ is the height of the Sun above the Galactic midplane according to the estimated given in [31].

\section{RESULTS AND DISCUSSION}

\section{Frequency of Globular-Cluster Impacts onto the Galactic Plane}

The MWSC provides the complete set of kinematic data for 133 GCs. This set includes the estimate of the distance to the GC, its sky coordinates, proper-motion components, radial velocity, and the measurement errors of these quantities. We used these and refined data (see "THE DATA" section) to integrate the Galactic orbit for each GC 1 billion years into the past.

To estimate the frequency of GC impacts onto the Galactic plane, we determine for each cluster the time instants when $Z=0 \mathrm{kpc}$. There are GCs, which have never reached the Galactic plane during the last one billion years. Most of the GCs have crossed the Galactic disk several times. Thus, e.g., $\omega$ Cen crossed the Galactic disk 26 times during one billion years. The record breaker is the GC VDBH229 (ESO 455-11), which is very close to the Galactic center and which crossed the Galactic disk 175 times during this time.

Fig. 1 shows the number of Galactic-plane crossings by the GCs of our sample as a function of the distance to the rotation axis of the Galaxy. The histograms shown in this figure are based on the data of two types: (a) the data adopted from the MWSC catalog and (b) the data refined as described in "THE DATA" section (the solid line). As is evident from 


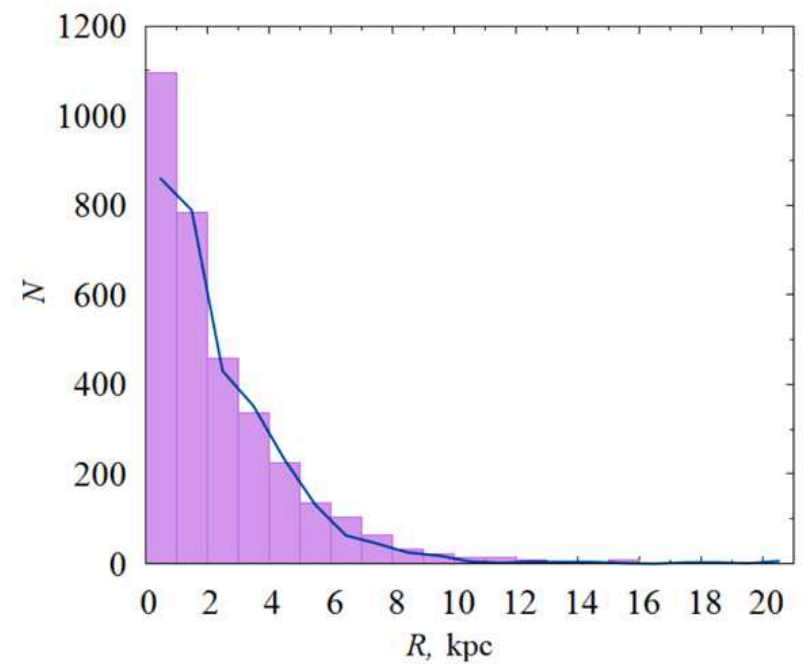

Figure 1: Histogram (the filled bars) of the number of the crossings of the Galactic plane $X Y$ by GCs during the last billion years based on the data of MWSC catalog [13]. The solid line shows the distribution based on refined data. The horizontal axis gives distance $R$ from the rotation axis of the Galaxy.

the figure, despite their scarcity, the use of refined data affected the domain in the vicinity of the Galactic center $(R<1 \mathrm{kpc})$.

A number of authors $[7,8]$ report, albeit without specifying the method employed, an estimate of the frequency of GC impacts onto the Galactic plane, which is of about one event in million years. We found that, on the average, three crossings occur in one million years within the entire disk.

When discussing the efficiency of the star-formation processes associated with the effect of GCs onto the Galactic disk, Salerno et al. [8] point out that effects of a GC impact onto the formation of OSCs in the local disk region (within 1-2 kpc) is highly likely in any place in the inner Galaxy about once every 10 million years. I.e., the above authors note the uniform nature of star formation in the disk region. We, however, may suggest, based on our inferred exponential decrease of the number of GC impacts onto the disk shown in Fig. 1, that the efficiency of star formation initiated by globular-cluster crossings of the Galactic disk also deceases exponentially with the distance from the Galactic center.

\section{Testing Two Well-Known Hypotheses}

Note that it takes considerable time after the GC impact onto the Galactic plane for an OSC to form. Following [7], we use the formula:

$$
t=t_{\mathrm{C}}+t_{\mathrm{SF}}+t_{\mathrm{A}}
$$

where $t$ is the time elapsed since the GC crossed the Galactic disk and until now; $t_{C}$, the time interval since the crossing time until the start of star formation; $t_{S F}$, the duration of star formation, and $t_{A}$, the age of the GC.

The value of the first term in formula (12) is known with large uncertainty and lies in the interval from 0 to $30 \mathrm{Myr}$. For example, according to the estimate reported in [32] and obtained by modeling the impact of a high-velocity cloud onto the disk, $t_{C}=15 \mathrm{Myr}$, 
whereas according to [4], this time interval is equal to $t_{C}=30 \mathrm{Myr}$. The second term is, according to [33], equal to $t_{\mathrm{SF}}=0.2 \mathrm{Myr}$ (for a star with the mass of $M>M_{\odot}$ ), which is small compared to other terms and hence this term can be neglected in the first coarse estimates.

We performed Monte-Carlo statistical simulations for each object studied (GC or OSC). We added normally distributed random errors with zero means and known standard deviations to the coordinates of the object $(X, Y, Z)$, its spatial velocities $(U, V, W)$, and parameters of the Gravitational potential model (see Table 1). We determined the velocities, coordinates, and their errors by applying Monte-Carlo statistical modelling method to measured heliocentric distances, proper motions, and radial velocities of the objects.

\section{The $\omega$ Cen-Stephenson 2 Pair}

Fig. 2 shows two diagrams obtained with two sets of measurement data for the GC $\omega$ Cen and OSC Stephenson 2, which we describe below. In these plots we show the (1) confidence intervals of the domain of points where the $\mathrm{GC} \omega$ Cen crossed the Galactic plane $X Y$ (the gray circles) obtained using Monte-Carlo method taking into account the measurement errors of the GC parameters and (2) the orbits of the OSC Stephenson 2 computed over the time interval from the present time to time $t$ of the GC crossing of the Galactic disk, also obtained using Monte-Carlo method. These plots give the idea of the degree of overlap between the set of points of the GC crossings of the Galactic disk and the set of end points of open-cluster trajectories at time $t$. If such sets overlap then we can conclude that the formation of the OSC could be triggered by the GC. In the absence of such overlap the conclusion is negative. Such an approach was implemented in the study of the other GC-OSC pairs described below.

We already discussed the parameter values for the GC $\omega$ Cen above in "THE DATA" section. Here we consider the parameters of the OSC Stephenson 2. According to estimates [34], the age of Stephenson 2 is equal to $t_{\mathrm{A}} \sim 20 \mathrm{Myr}$ and it is located at heliocentric distance of $d=5.9 \mathrm{kpc}$. They are also consistent with more recent estimates [35]: the age is in the $t_{A}=12-17 \mathrm{Myr}$ interval and the distance is $d=5.8_{-0.8}^{+1.9} \mathrm{kpc}$. It is important that Davies et al. [35] measured radial velocities for 26 stars of the OSC Stephenson 2 and determined the average radial velocity of the OSC $V_{\mathrm{LSR}} \approx 110 \pm 4 \mathrm{~km} \mathrm{~s}^{-1}$, which we used together with the heliocentric distance $d=5.8 \pm 0.6 \mathrm{kpc}$ for analyzing the $3 \mathrm{D}$ motion of this cluster. We combined these with two sets of proper motion values for Stephenson 2:

$$
\begin{array}{r}
\mu_{\alpha} \cos \delta=2.69 \pm 1.43{\operatorname{mas~} \mathrm{yr}^{-1}} \\
\mu_{\delta}=-7.00 \pm 1.43{\operatorname{mas~} \mathrm{yr}^{-1}}
\end{array}
$$

according to [13] and

$$
\begin{array}{r}
\mu_{\alpha} \cos \delta=-0.44 \pm 1.21{\operatorname{mas~} \mathrm{yr}^{-1}} \\
\mu_{\delta}=0.80 \pm 0.53 \mathrm{mas} \mathrm{yr}^{-1}
\end{array}
$$

according to catalog [36]. In catalog [36] the average proper motion of the OSC are based

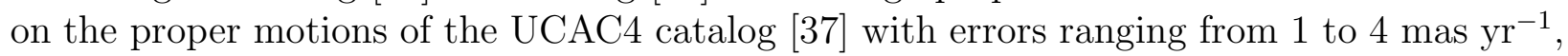
which are substantially smaller than the corresponding errors in the PPMXL catalog [15].

As we already pointed out above, Fig. 2 shows the results obtained for two sets of data used to analyze the $\omega$ Cen-Stephenson 2 pair. We refer to the data combination (2) for $\omega$ Cen and (13) for Stephenson 2 as the "old" data and the combination (1) and (14) as the "new" data. 


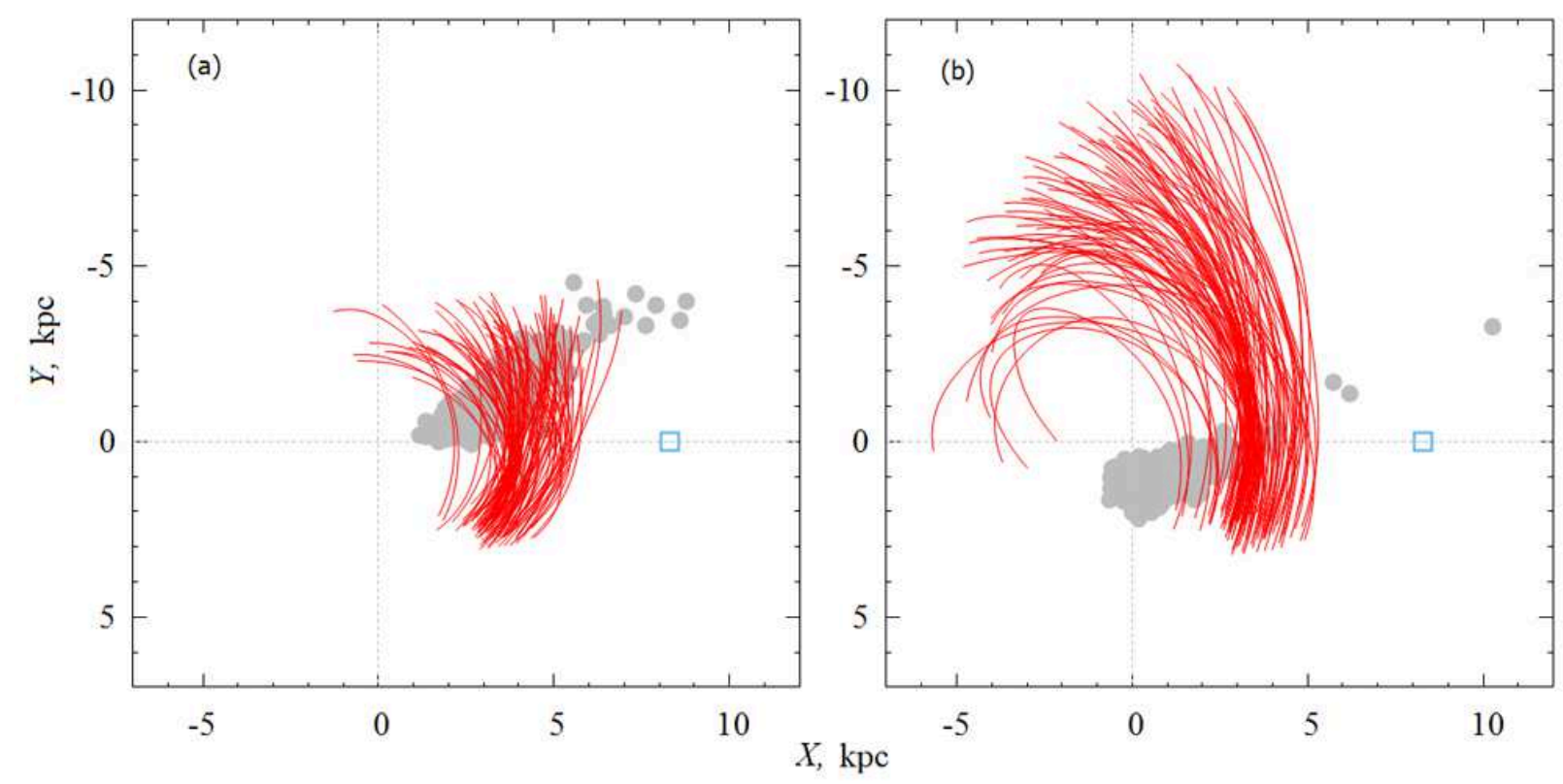

Figure 2: Confidence intervals of the intersection of $\omega$ Cen GC with the Galactic plane $X Y$ (the gray circles) and ensembles of orbits of the OSC Stephenson 2, (the solid lines) obtained using Monte-Carlo method over the time interval from the last GC crossing of the Galactic disk to the present time based on "old" (a) and "new" (b) data. The Galactic center is located at the origin and the square indicates the position of the Sun. Currently, the Stephenson 2 cluster is located in the first Galactic quadrant.

Note that our approach differs from that of Salerno et al. [8] in that we computed the spatial velocities of Stephenson 2 using real measurements of both the proper motions and radial velocity. The above authors [8] computed the model velocities of Stephenson 2 assuming that it moves in a purely circular orbit about the Galactic center.

Based on the "old" data we found that $\omega$ Cen crossed the Galactic plane 27.5 million years ago. This is the nominal value and it is close to the $t=24 \mathrm{Myr}$ estimate obtained in [8]. Given that the age of Stephenson 2 is $t_{A}=20 \mathrm{Myr}$ [34] we can conclude that the hypothesis of Salerno et al. [8] that the formation of this OSC may have been initiated by the impact of the GC $\omega$ Cen onto the Galactic disk is true if it took the OSC 7.5 Myr to form in accordance with formula (12). Fig. 2a serves as a weak confirmation of this hypothesis: the set of the end points of the open-cluster trajectories corresponding to time $t=27.5 \mathrm{Myr}$ overlaps, albeit only slightly, with the domain of the globular-cluster crossings points with the Galactic disk. In this case the nominal distance from the OSC orbit to the GC at the time of the disk crossing was $2.49 \mathrm{kpc}$ (see Table 2).

The situation is radically different if we use the "new" data set for the GC $\omega$ Cen and OSC Stephenson 2. In this case the nominal estimate of the time of crossing of the disk by the GC is $t=45.5 \mathrm{Myr}$ and we integrate the orbits of the OSC backwards over this time. As is evident from Fig. 2b, the end points of the orbits of the OSC Stephenson 2 pass rather far from the domain of intersection of the GC $\omega$ Cen with the $X Y$ plane: the nominal distance between the open-cluster orbit and the GC at the time of the disk crossing is $9.59 \mathrm{kpc}$ (see Table 2). We can conclude from this that the hypothesis of Salerno et al. [8] is strongly inconsistent with the new data about the GC $\omega$ Cen and OSC Stephenson 2. 


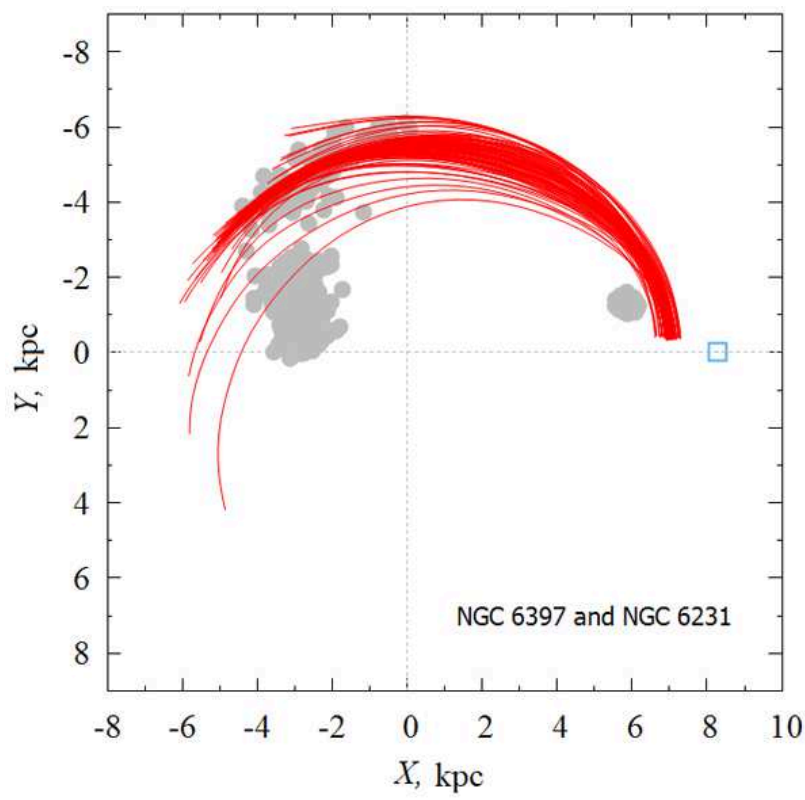

Figure 3: Confidence domains of two successive crossings of the Galactic plane $X Y$ by the GC NGC6397 (the gray circles) and the ensemble of orbits (the solid lines) of the OSC NGC6231 constructed over the 49.5 Myr time interval backwards using Monte-Carlo method. In the righthand part of the figure we show the confidence domains of the last crossing of the GC (3.5 million years ago), and in the left-hand part, the confidence domains of the previous crossing (49.5 million years ago). The Galactic center is at the origin and the square indicates the position of the Sun. The OSC NGC6231 is currently located in the second Galactic quadrant near the Sun.

It is interesting that according to [13], the age of Stephenson 2 is $t_{A}=1$ Myr. By combining this age estimate with the "new" data set we obtain too large time difference $t-t_{A}=44.5 \mathrm{Myr}$, which is far beyond our adopted interval. This fact further strengthens our conclusion that pairing these two clusters is wrong.

\section{The NGC6397-NGC6231 Pair}

According to the hypothesis of Rees and Cudworth [6], the passage of the GC NGC6397 through the Galactic plane could trigger the formation of the OSC NGC6231. The GC NGC6397 is also included into list [7] as a candidate object for searching for any manifestations of its Galactic-plane crossing. The above authors analyzed the possibility of the formation of the OSC NGC6231 and concluded that because of its young age $\left(t_{A}=3-5 \mathrm{Myr}\right)$ and quite large distance from the site of the GC impact onto the disk it could not form as a result of the passage of the GC NGC6397 through the Galactic plane, which, according to their estimate, occurred 3.7 million years ago.

Our analysis of this pair led us to the same conclusion. The age of the OSC NGC6231 is equal to $t_{A}=11.2 \mathrm{Myr}$ [13], and the last time when the GC NGC6397 crossed the Galactic plane was about 3.5 million years ago. In this case condition (12) is evidently not fulfilled.

Although the projections of the trajectories of the Galactic orbits of the two clusters onto the $X Y$ plane are close to each other, they can be quite far away from each other in the 3D space. Indeed, the vertical velocity $W=-4.3 \pm 0.5 \mathrm{~km} \mathrm{~s}^{-1}$ of the OSC NGC6231 is small and therefore its orbit lies practically in the Galactic plane. The corresponding velocity 
component of the GC NGC6397 is much greater and equal to $W=-131.8 \pm 6.7 \mathrm{~km} \mathrm{~s}^{-1}$, and therefore its orbit rises high above the Galactic plane (i.e., the GC may go far away from the OSC NGC6231 along the $Z$-axis). Note the that random errors of the kinematical properties of both clusters are small enough.

As we pointed out in "THE DATA" section, the proper motion of the NGC6397 were computed by Watkins and van der Marel [18] based on the data from Gaia TGAS catalog, i.e., they are sufficiently reliable. The trigonometric parallax of the cluster was determined by Brown et al. [20] with a fractional error of about 4\%. As a result, statistical simulations produce compact confidence domains for crossings of this GC.

After constructing the Galactic orbits of these two clusters over the 50-Myr time interval backwards we found surprisingly that they approach the GC at the time of the previous Galactic-plane crossing 49.5 Myr ago to a nominal distance of about $3.55 \mathrm{kpc}$ (see Table 2, which gives the approach parameters both for the last (case I) and the second last (case II) crossings). Fig. 3 shows the confidence domains of the points of the crossing of the Galactic plane $X Y$ by the GC NGC6397 and the trajectory of the OSC NGC6231 constructed over the 49.5 Myr interval backwards with the allowance for data errors found via Monte-Carlo statistical modeling method. In the right-hand part of the figure the gray circles fill the domain of the last crossing of the Galactic disk by the GC, which occurred 3.5 million years ago, and in the left-hand part of the figure the gray circles show the domain of the second-last crossing that occurred 49.5 million years ago. One can see in the figure the overlap of the end points of the open-cluster orbits and the domain of the previous Galactic-disk crossing by the GC. This fact indicates that the effect of the GC NGC6397 could, in principle, have triggered the formation of the OSC NGC6231 about 49.5 million years ago during the previous GC crossing of the Galactic plane. However, in this case the time interval from the crossing to the start of star formation, $t-t_{A}=38 \mathrm{Myr}$, should have been unrealistically long. This fact leads us to conclude that the NGC6397(II)-NGC6231 scenario is also highly unlikely.

\section{Search for New Candidates}

We the searched for new candidate pairs of the form GC-OSC. To this end, we composed, in addition to the already available sample of $133 \mathrm{GCs}$, a sample of young $(\log t<7.6)$ OSCs with measured proper motions, radial velocities, and distances. This sample included 200 OSCs distributed over the entire Galactic disk. For each pair we computed the so called encounter parameter $\Delta r_{t}$, which is actually equal to the distance between the globular and OSC at time $t$, when the GC crossed the Galactic plane.

The results of the search obtained subject to the constraints mentioned below are listed in Table 2. Note that the first four rows of the table give the parameters of the two GC-OSC pairs considered above. The second (bottom) part of the table presents the new results.

We impose the following constraints: (1) $\Delta r_{t}$, should not exceed $20 \%$ of the heliocentric distance of the GC to the crossing place (this constraint is based on the random error of photometric distance), (2) the evident condition $t_{A}<t$ should be satisfied in accordance with formula (12). It follows from Table 2 that even with such conditions we have several open cluster candidates for some GCs.

For the new candidate GCs we considered the OSCs associated with the last crossing of the Galactic disk by the GC, and for the GC NGC6749 we considered the OSCs associated with the place of the second-last (II) crossing, which is more appropriate for the formation of the OSC in terms of the temporal parameters. 
Table 2: Parameters of the candidate GC-OSC pairs for past encounters that we found in this study

\begin{tabular}{|l|l|c|c|c|c|}
\hline GC & OSC & $\begin{array}{c}\Delta r_{t}, \\
\mathrm{kpc}\end{array}$ & $\begin{array}{c}t, \text { Myr } \\
\text { in the past }\end{array}$ & $\begin{array}{c}t_{A}, \\
\mathrm{Myr}\end{array}$ & $\begin{array}{c}t-t_{A}, \\
\mathrm{Myr}\end{array}$ \\
\hline$\omega$ Cen (old data) & Stephenson2 & 2.49 & 27.5 & 20.0 & 7.5 \\
$\omega$ Cen (new data) & Stephenson2 & 9.59 & 45.5 & 20.0 & 25.0 \\
NGC 6397 (I) & NGC 6231 & 0.96 & 3.5 & 11.2 & -7.7 \\
NGC 6397 (II) & NGC 6231 & 3.55 & 49.5 & 11.2 & 38.3 \\
\hline NGC 104 & Ruprecht 129 & 0.25 & 52.5 & 31.6 & 20.9 \\
NGC 104 & FSR 52 & 0.52 & 52.5 & 31.6 & 20.9 \\
NGC 104 & Ruprecht 127 & 0.96 & 52.5 & 35.5 & 17.0 \\
NGC 104 & NGC 6396 & 0.90 & 52.5 & 32.0 & 20.5 \\
NGC 104 & Trumpler 27 & 1.44 & 52.5 & 38.0 & 14.5 \\
NGC 2808 & Pismis 20 & 1.39 & 41.5 & 31.6 & 9.9 \\
NGC 2808 & IRAS 6567 & 2.50 & 41.5 & 22.4 & 19.1 \\
NGC 2808 & Haffner 16 & 2.16 & 41.5 & 20.0 & 21.5 \\
NGC 6362 & Pismis 11 & 0.55 & 21.5 & 1.0 & 20.5 \\
NGC 6540 & [DBS2003] 102 & 0.57 & 15.5 & 1.3 & 14.2 \\
NGC 6541 & [DBS2003] 161 & 0.63 & 26.5 & 1.0 & 25.5 \\
NGC 6541 & [DBS2003] 102 & 1.23 & 26.5 & 1.3 & 25.2 \\
NGC 6749 (II) & Trumpler 27 & 1.23 & 51.5 & 38.0 & 13.5 \\
NGC 6749 (II) & Ruprecht 127 & 1.41 & 51.5 & 35.5 & 16.0 \\
NGC 6749 (II) & Turner 3 & 1.77 & 51.5 & 28.8 & 23.3 \\
NGC 6749 (II) & NGC 6396 & 1.88 & 51.5 & 32.0 & 19.5 \\
NGC 6749 (II) & Ruprecht 129 & 1.96 & 51.5 & 31.6 & 19.9 \\
NGC 6749 (II) & FSR 52 & 2.26 & 51.5 & 31.6 & 19.9 \\
NGC 6752 & [DBS2003] 115 & 0.90 & 47.5 & 24.0 & 23.5 \\
\hline
\end{tabular}




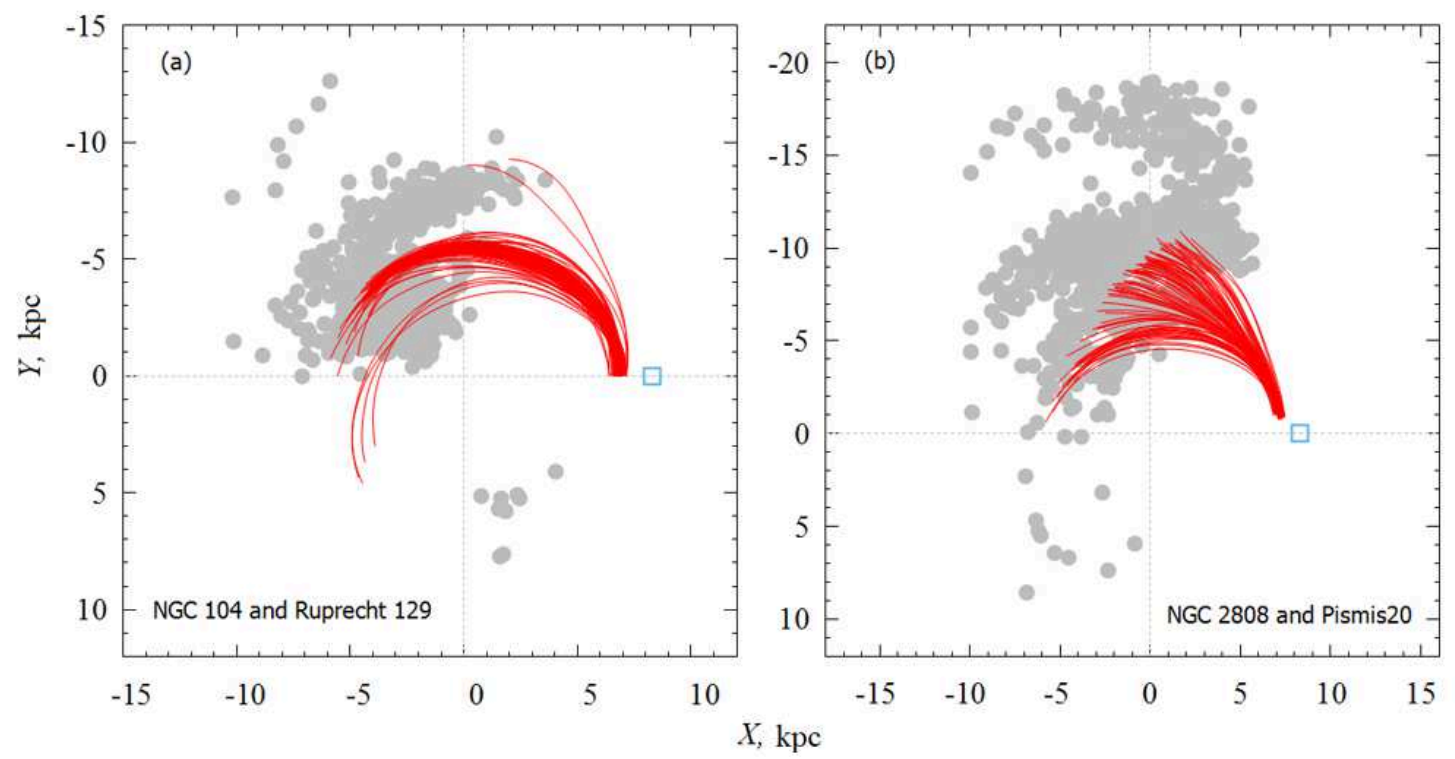

Figure 4: Confidence domains of the GC crossing points with the Galactic plane $X Y$ (the gray circles) and ensembles of open-cluster orbits (the solid lines): (a) for the NGC104-Ruprecht 129 pair over the 52.5-Myr backward time interval obtained using Monte-Carlo method, (b) for the NGC2808-Pismis 20 pair over the 41.5-Myr backward time interval. The Galactic center is at the origin and the square denotes the position of the Sun.

Figs. 4-6 illustrate for a number of selected GC-OSC pairs the degree of overlap between the confidence domains of the crossings of the Galactic disk by the GC and the end points of the open cluster orbits corresponding to the impact time. An analysis of the modeling results obtained suggests that the birth of the OSC could have been triggered by the GC. Below we give comments about individual objects.

\section{NGC 104}

The GC NGC104 is located sufficiently close to the Sun $(d=4.50 \pm 0.05 \mathrm{kpc})$ and its kinematical properties are well known. For this cluster we use the proper motion computed in [18] based on Gaia TGAS catalog. The mass of NGC104 is $0.84 \times 10^{6} M_{\odot}$ [38], which is more than the masses of most of the Galactic GCs with known mass estimates $(1-3) \times 10^{5} M_{\odot}$ $[7]$.

Fig. 4a shows the confidence domains of the NGC104 globular-cluster crossing points with the Galactic plane $X Y$ and trajectories of the OSC Ruprecht 129 computed over the 52.5-Myr backward time interval. As is evident from the figure, we have reliable overlap between the regions of the GC crossings of the Galactic plane and the end points of the orbits of the OSCs considered, providing a strong case for the hypothesis that the formation of the OSC was triggered by the passage of the GC across the Galactic disk.

Vande Putte and Cropper [7] determined for a number of GCs the expected positions of OSCs at present assuming that their formation was triggered by the last passage of the GC through the disk. They give confidence intervals for each such position and, as direct comparison of the positions has shown, all the OSCs listed in Table 2 are either inside or close to the confidence domain found in [7] for the GC NGC104. It is interesting that Vande Putte 


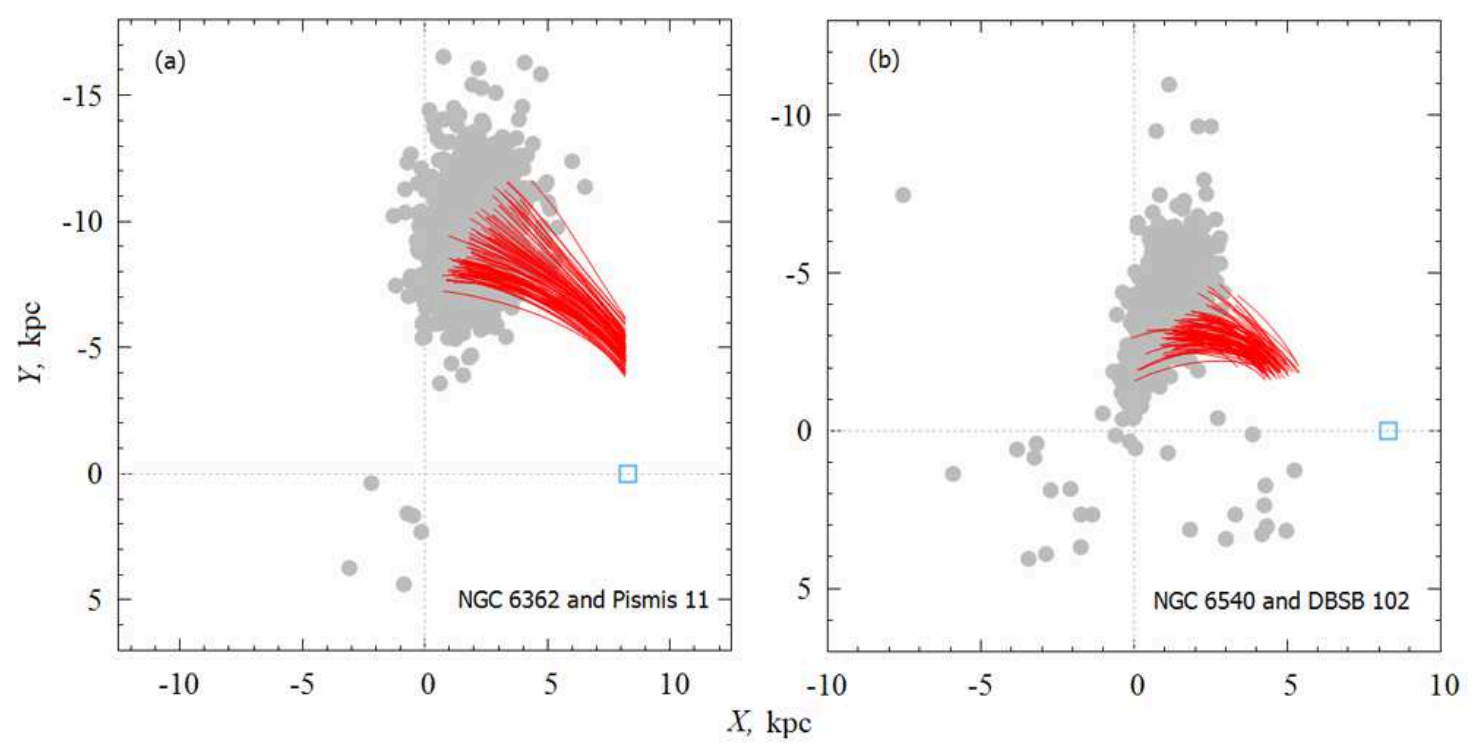

Figure 5: Confidence domains of the GC crossing points with the Galactic plane $X Y$ (the gray circles) and ensembles of open-cluster orbits (the solid lines): (a) for the NGC6362-Pismis 11 pair over the 21.5-Myr backward time interval obtained using Monte-Carlo method, (b) for the NGC6540-[DBS2003] 102 pair over the 15.5-Myr backward time interval. The Galactic center is at the origin and the square denotes the position of the Sun.

and Cropper [7] used several models of the Galactic gravitational potential. For example, in the Flynn potential model (they denote this model as FL) they found for NGC104 the time of the last Galactic plane crossing to be $t=52.6 \pm 1.9 \mathrm{Myr}$, which agrees well with our crossing time of $t=52.5 \mathrm{Myr}$.

The coordinates of all OSCs mentioned in Table 2 are close to each other. However, the pair of OSCs with exactly the same ages-Ruprecht 129 and FSR52-stands out and therefore. Therefore it is appropriate to raise the question about their simultaneous origin.

\section{NGC 6352 and NGC 6362}

Fig. 5a illustrates the case of the NGC6362-Pismis 11 pair. All parameters appear to be acceptable for this pair: we are dealing with a rather close encounter $\Delta r_{t}=0.55 \mathrm{kpc}$ and realistic time interval $t-t_{A}=20.5 \mathrm{Myr}$. As is evident from the figure, all end points of open-cluster trajectories are located inside the confidence domain of the GC crossing points, which, in addition, is more compact compared to other cases. From this viewpoint the NGC6362-Pismis 11 pair is one of the best candidates in our list.

Fig. 5b illustrates the case of the NGC6540-[DBS2003] 102 pair. It stands out by its compact domain of crossing points and the fact that practically $100 \%$ of the end points of the open-cluster trajectories are located inside this domain. The pair underwent a rather close encounter, $\Delta r_{t}=0.57 \mathrm{kpc}$, and is characterized by rather short time interval $t-t_{A}=$ 14.2 Myr. 

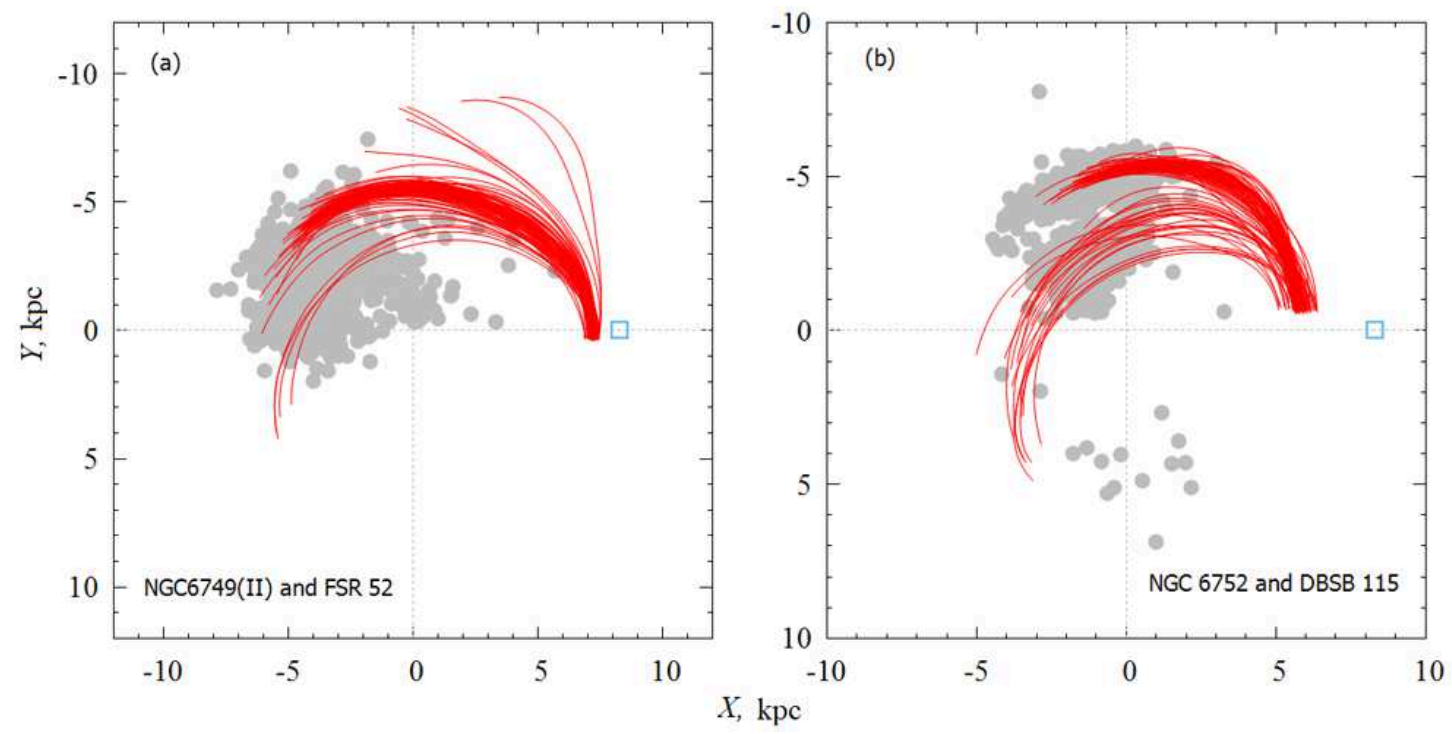

Figure 6: Confidence domains of the GC crossing points with the Galactic plane $X Y$ (the gray circles) and ensembles of open-cluster orbits (the solid lines): (a) for theNGC6749(II)-FSR52 pair over the 51.5-Myr backward time interval obtained using Monte-Carlo method, (b) for the NGC6752-[DBS2003] 115 pair over the 47.5-Myr backward time interval. The Galactic center is at the origin and the square denotes the position of the Sun.

\section{NGC6749 and NGC6752}

A noteworthy feature of the GC NGC6749 is that almost all its purportedly associated OSCs listed in Table 2 came close not only to the place of the second-last crossing, but also to the place of the last Galactic-disk crossing of the GC NGC104.

Fig. 6a illustrates the case of the NGC6749 (II)-FSR52, and Fig. 6b, that of the NGC6752-[DBS2003] 115 pair. Both of these pairs are characterized by low probability, of about $50 \%$, of open-cluster trajectories getting into the domain of GC crossing points.

\section{CONCLUSIONS}

We computed the Galactic orbits of 133 GCs by integrating their trajectories in the Galactic gravitational potential that we earlier refined based on modern data.We obtained a new estimate for the frequency of globular-cluster impacts onto the Galactic plane, which is equal to three events in 1 million years. We showed that the number of such events is distributed exponentially with a maximum in the central part of the Galaxy and rapidly declines with the distance from the Galactic center. One should therefore expect the efficiency of star formation initiated by "bombardment" of the Galactic disk by GCs should also depend strongly on Galactocentric distance. Another conclusion that follows from it is that it is more efficient to search for possible GC-OSC pairs in the inner regions of the Galaxy, and such a search will be more resultative after the completion of the Gaia space mission [39].

We tested two well-known hypotheses about the formation of an OSC as a result of a Galactic-disk crossing by a GC.

The use of modern kinematical data did not support the hypothesis of Salerno et al. [8] 
that the OSC Stephenson 2 could form after the passage of the massive GC $\omega$ Cen through the Galactic disk.

The hypothesis of Rees and Cudworth [6] that the passage of the GC NGC6397 through the Galactic plane could have triggered the formation of the OSC NGC6231 could be true not for the last Galactic-disk crossing of NGC6397, which occurred 3.5 million years ago as the authors of the hypothesis believed, but for the previous passage, which occurred about 49.5 million years ago. However, this would require unrealistically long time interval between the crossing and the beginning of star formation, $t-t_{A}=38 \mathrm{Myr}$. This leads us to conclude that the scenario of Rees and Cudworth [6] is unlikely.

We searched for new GC-OSC candidate pairs using the sample of 133 GCs and 200 OSCs and assessing the GC-OSC encounters by analyzing each such pair. As a result, we identified six GCs indicating for each one of them one or several OSCs potentially paired with them (we list all these clusters in Table 2) within the framework of the scenario where the formation of an OSC could have been caused by the passage of a massive GC through the Galactic disk. These GCs are NGC104, NGC2808, NGC6362, NGC6540, NGC6749, and NGC6752. We found the pair with the closest distance between the GC and OSC at the time of the Galactic disk crossing by the GC to be NGC104-Ruprecht 129, which also has compatible temporal parameters. Thus it is likely that the Galactic-disk crossing of the GC NGC104 52.5 Myr ago could have served as the mechanism that initiated star formation at the crossing site. After 20.9 Myr the OSC Ruprecht 129 formed whose age is estimated at 31.6 Myr. Also noteworthy is the GC-OSC pair NGC6362-Pismis11, which has consistent parameters for the realization of a similar scenario.

\section{ACKNOWLEDGMENTS}

We are grateful to the reviewer for useful comments, which helped to improve the paper. This work was supported by Program P-28 of the Presidium of the Russian Academy of Sciences, subprogram "Space: the study of fundamental processes and their interactions".

\section{REFERENCES}

1. F. Comeron and J. Torra, Astron. and Astrophys. 261, 94 (1992).

2. F. Comeron and J. Torra, Astron. and Astrophys. 281, 35 (1994).

3. V. V. Levy, Astron. Astrophys. Transactions 18, 621 (2000).

4. J. F. Wallin, J. L. Higdon, and L. Staveley-Smith, Astrophys. J. 459, 555 (1996).

5. P. Brosche, H.-J. Tucholke, A. R. Klemola, et al., Astron. J. 102, 2022 (1991).

6. R. F. Rees and K. M. Cudworth, BAAS 35, 1219 (2003).

7. D. Vande Putte and M. Cropper, Monthly Notices Royal Astron. Soc. 392, 113 (2009).

8. G. M. Salerno, E. Bica, C. Bonatto, and I. Rodrigues, Astron. and Astrophys. 498, 419 (2009).

9. R. de la Fuente Marcos, C. de la Fuente Marcos, and D. Reilly, Astrophys. and Space Sci. 349,379 (2014).

10. D. Massari, A. Bellini, F. R. Ferraro, et al., Astrophys. J. 779, 81 (2013).

11. L. J. Rossi, S. Ortolani, B. Barbuy, et al., Monthly Notices Royal Astron. Soc. 450, 3270 (2015).

12. M. Libralato, A. Bellini, L. R. Bedin, et al., Astrophys. J. 854, 45 (2018).

13. N. V. Kharchenko, A. E. Piskunov, E. Schilbach, et al., Astron. and Astrophys. 558, A53 (2013). 
14. N. V. Kharchenko, A. E. Piskunov, E. Schilbach, et al., Astron. and Astrophys. 585, A101 (2016).

15. S. Roeser, M. Demleitner, and E. Schilbach, Astron. J. 139, 2440 (2010).

16. V. V. Bobylev and A. T. Bajkova, Astronomy Reports 61, 551 (2017).

17. A. Pérez-Villegas, L. Rossi, S. Ortolani, et al., Publ. Astron. Soc. Australia 35, e021 (2018).

18. L. L. Watkins and R. P. van der Marel, Astrophys. J. 839, 89 (2017).

19. D. Massari, L. Posti, A. Helmi, et al., Astron. and Astrophys. 598, L9 (2017).

20. T. M. Brown, S. Casertano, J. Strader, et al., Astrophys. J. 856, L6 (2018).

21. A. T. Bajkova and V. V. Bobylev, Astronomy Letters 42, 567 (2016).

22. A. Bajkova and V. Bobylev, Open Astronomy 26, 72 (2017).

23. J. F. Navarro, C. S. Frenk, and S. D. M. White, Astrophys. J. 490, 493 (1997).

24. M. Miyamoto and R. Nagai, Publ. Astron. Soc. Japan 27, 533 (1975).

25. J. Palous, B. Jungwiert, and J. Kopecky, Astron. and Astrophys. 274, 189 (1993).

26. V. V. Bobylev and A. T. Bajkova, Astronomy Letters 42, 228 (2016).

27. C. C. Lin and F. H. Shu, Astrophys. J. 140, 646 (1964).

28. C. C. Lin, C. Yuan, and F. H. Shu, Astrophys. J. 155, 721 (1969).

29. D. Fernández, F. Figueras, and J. Torra, Astron. and Astrophys. 480, 735 (2008).

30. R. Schö nrich, J. Binney, and W. Dehnen, Monthly Notices Royal Astron. Soc. 403, 1829 (2010).

31. V. V. Bobylev and A. T. Bajkova, Astronomy Letters 42, 1 (2016).

32. J. R. D. Lepine and G. Duvert, Astron. and Astrophys. 286, 60 (1994).

33. C. F. McKee and J. C. Tan, Nature 416, 59 (2002).

34. S. Ortolani, E. Bica, B. Barbuy, and Y. Momany, Astron. and Astrophys. 390, 931 (2002).

35. B. Davies, D. F. Figer, R.-P. Kudritzki, et al., Astrophys. J. 671, 781 (2007).

36. W. S. Dias, H. Monteiro, T. C. Caetano, et al., Astron. and Astrophys. 564, A79 (2014).

37. N. Zacharias, C. T. Finch, T. M. Girard, et al., Astron. J. 145, 44 (2013).

38. A. Bellini, P. Bianchini, A. L. Varri, et al., Astrophys. J. 844, 167 (2017).

39. Gaia Collab., T. Prusti, J. H. J. de Bruijne, et al., Astron. and Astrophys. 595, A1 (2016). 